\title{
El ETM de Futuros Profesores de Primaria en un Trabajo sobre los Números Naturales en Cualquier Base
}

\author{
Future Elementary School Teachers' Mathematical Working Space for \\ Working with Natural Number in Any Base
}

\author{
Kostas Nikolantonakis ${ }^{*}$ \\ Laurent Vivier $^{* *}$
}

\begin{abstract}
Resumen
La formación de profesores de primaria, en Francia como en Grecia, pone el acento en los números naturales y el sistema posicional. La introducción de bases diferentes de diez muestra que los Espacio de Trabajo Matemático (ETM) personales son guiadados por el componente semiótico (representamen) reducido a la base diez y que una parte del trabajo se puede interpretar como un cambio de dominio, entre la base diez y las otras bases. Proponemos retomar los análisis semióticos y estadísticos de una experimentación realizada en Francia y en Grecia con el modelo de los ETM que permite tomar en cuenta los diferentes componentes del trabajo matemático. El estudio finaliza con una experimentación elaborada en base siete que parece ayudar a la conceptualización y a la coordinación de los ETM relativos a los números en diferentes bases.
\end{abstract}

Palabras clave: Espacio de Trabajo Matemático. Números Naturales. Sistema Posicional. Formación Inicial de Docentes.

\begin{abstract}
Primary school teachers' training in France and Greece emphasizes natural numbers and the positional system. The introduction of bases other than ten shows that personal ETMs are guided by semiotic component (representamen) reduced to the ten base and that some of the work can be interpreted as a change of domain between the ten base and the other bases. This work will resume the semiotic, praxeological and statistical analysis conducted for an experiment in France and Greece using the Mathematical Working Space (MWS) model, which allows us to take into account the different components of the mathematical work. The study was completed by an experiment developed on a seven base that seemed to help in the conceptualization and coordination between the MWS related to the different bases.
\end{abstract}

Keywords: Mathematical Working Space. Natural Numbers. Positional System. Teachers' Initial Training.

\footnotetext{
* Doctor en Historia y Epistemología de Matemáticas de la Universidad Paris-7 Denis Diderot, 5 rue Thomas Mann 75205 Paris cedex 13 Francia; Profesor Asociado de la Universidad de Macedonia Oeste, 3e Km Florina Niki, 53100, Florina, Grecia, nikolantonakis@ noesis.edu.gr.

** Doctor en matemática de la universidad de Tours, 60 rue du Plat D'Etain 37020 Tours Cedex 1 Francia; Habilitado a Dirigir Investigaciones (HDR) en Didáctica de las Matemáticas de la Universidad Paris Diderot, 5 rue Thomas Mann 75205 Paris cedex 13 Francia; Profesor Asociado en el LDAR, Universidad de Paris Diderot Case 7018- Place Aurélie Nemours Bâtiment Sophie Germain 75205 Paris Cedex 13 Francia, y en el IMAG, Universidad de Montpellier, -Case 051- Place Eugène Bataillon 34095 Montpellier Cedex 5 Francia; laurent.vivier@univ-paris-diderot.fr.
} 


\section{Introducción}

El dominio numérico es una parte esencial de las matemáticas. Su enseñanza comienza desde la escuela maternal y continua durante toda la escolaridad, enriqueciéndose sin cesar de variedad de números o de nuevos puntos de vistas teóricos. En este contexto, la posición de la numeración que utilizamos es fundamental porque una gran parte de lo numérico se apoya sobre ella. Ahora bien, este sistema posicional de representación de los números es complejo porque condensa en pocos signos numerosos significados y los alumnos tienen dificultades en su aprendizaje, aprendizaje que se limita a la base diez.

En este contexto, y aunque sea no específico matemáticamente, comprendemos por qué la formación inicial y continua de profesores se interesa en el dominio numérico y a la numeración posicional decimal.

Sin embargo, aunque los futuros profesores solo tendrán que enseñar la numeración posicional en base diez, en la formación inicial la numeración en otras bases que la diez es utilizada para sensibilizar a los futuros profesores en las dificultades que sus alumnos podrían tener. Adicionalmente, les permite desnaturalizar sus conocimientos ${ }^{1}$ que pueden ser un obstáculo en la enseñanza, darse cuenta del carácter relativo de la base diez y entender los mecanismos generales de la numeración posicional. Sin embargo, los futuros profesores saben lo esencial de la base diez. Así, para tareas vinculadas a estos conocimientos, dos fenómenos pueden ser considerados en la formación de profesores:

1. Una conversión en base diez para hacer las tareas inicialmente formuladas en otra base. En este caso, podemos pensar que no alcanzamos el objetivo de formación ya que el nuevo sistema de representación de los números no puede desarrollarse (ver a NIKOLANTONAKIS; VIVIER, 2010; 2013, y la sección 3); el sistema cumple solo la función de comunicación y no se constituye como un registro ya que no cumple las otras dos funciones cognitivas de tratamiento y objetivación (DUVAL, 1996).

2. Una utilización de las propiedades de la base diez en otras bases, sin control ni justificación, para hacer las tareas en la base inicial. La proximidad de las reglas de representación de los registros constituidos por dos bases diferentes -así como de

\footnotetext{
${ }^{1}$ Podemos particularmente pensar en la asociación natural entre 5 , cinco y $\because \because$ o entre 12 , doce y $\because \because \because \because$. Se puede remitir al estudio de Anselmo, Dussuc y Zucchetta (2013) que propone una secuencia oral para la base seis cuyas cifras van desde las letras A a la F.
} 
algunos tratamientos- favorece ampliamente la utilización de las propiedades de la base diez en otras bases. Aunque el trabajo se realice de manera interna, en el registro de una base diferente a la de diez, sin control ni justificación las nuevas propiedades en el nuevo registro puede ser erróneas o sin sentido para el estudiante.

En ambos casos, no parecen ser alcanzados los objetivos de conceptualización esperada y de enriquecimiento del Espacio de Trabajo Matemático (ETM) personal del futuro profesor con los números naturales por la integración de nuevos registros. En el fenómeno 1, al incorporar nuevos registros no hay desarrollo ni evolución del referencial teórico, y en el fenómeno 2, el referencial teórico se desarrolla pero por una importación sin control, y a veces errónea, de propiedades de la base diez.

Después de una presentación del modelo de los ETM, en relación al número natural, presentamos en la sección 3 un estudio ${ }^{2}$ en la formación de profesores en Francia y en Grecia. Este estudio es una continuidad del trabajo entre ambos países. En este tema de los números naturales, con los dos fenómenos, como por el tema de la geometría (NIKOLANTONAKIS; VIVIER, 2015), los resultados muestran una diferencia entre el trabajo de los dos poblaciones al respecto de la flexibilidad de los saberes. Los análisis utilizan, nuevamente, el modelo de los ETM (KUZNIAK, 2011).

En la sección 4, utilizamos las ideas de la tesis de Tempier (2013) para elaborar una situación de descubrimiento de la numeración posicional en base siete que podría permitir un desarrollo más equilibrado del ETM en juego, es decir, tomando en consideración el referencial teórico. La situación será aplicada, con adaptaciones, en Grecia en 2016 cuyo objetivo es también comparar los resultados obtenidos a los estudios anteriores.

\section{Marco del estudio}

En esta sección, describimos el modelo de los ETM, y después las características del ETM de los números naturales en juego en nuestro estudio.

\subsection{El modelo de los ETM}

2 Este estudio fue analizado con el Análisis Estadístico Implicativo y con praxeologías, ver (NIKOLANTONAKIS; VIVIER, 2010, 2013). 
El ETM contiene dos planos (KUZNIAK, 2011), uno de naturaleza epistemológica y otro de naturaleza cognitiva. El plano epistemológico contiene el artefacto, el referencial teórico y el representamen (las representaciones semióticas). El plano cognitivo contiene los procesos de visualización, construcción y prueba.

El trabajo matemático en un marco escolar podrá ser descrito gracias a tres tipos de ETM. La matemática esperada por la institución se describe en el ETM de referencia. Este último debe ser elaborado por el profesor en un ETM idóneo para permitir una utilización efectiva en las aulas, donde cada alumno trabaja con su ETM personal.

La génesis global del ETM supone un conjunto de génesis que son interdependientes y que conciernen a todas las componentes del plano epistemológico y del plano cognitivo:

1. La génesis instrumental que permite que el artefacto sea operatorio en el proceso de construcción;

2. La génesis semiótica que da un sentido a los objetos del ETM y les confiere su estatuto de objetos matemáticos operatorios;

3. La génesis discursiva de la prueba que da un sentido a las propiedades.

Los planos verticales (KUZNIAK; RICHARD, 2014) introducidos en el esquema son relacionados a las diferentes fases del trabajo matemático en la ejecución de una tarea. El primer tipo de interacciones (plano Semiótico-Instrumental [Sem-Ins]) privilegia la identificación y la exploración de los objetos, apoyándose en los génesis semiótica e instrumental, para desarrollar la competencia vinculada al descubrimiento en la solución de problemas matemáticos. Un segundo tipo de interacciones (plano Instrumental-Discursivo [Ins-Dis]) desarrolla el razonamiento matemático fundado sobre la justificación de los descubrimientos, articulando las génesis instrumental y discursiva. El último tipo (plano Semiótico-Discursivo [Sem-Dis]) está orientado hacia la comunicación matemática de los resultados, y esencialmente se apoya en las génesis semiótica y discursiva.

\subsection{La cuestión de los paradigmas}

Al contrario de la geometría (KUZNIAK, 2006) o en el análisis (MONTOYA; VIVIER, trabajo en revisión), en el ETM del número natural, la aritmética elemental, la noción de paradigma desempeña un papel secundario. No hay diferencia, por ejemplo, entre una prueba formal con " $n$ " o por extensión en un cálculo explícito para todos los números. En geometría, ver dos rectas perpendiculares y mostrar que dos rectas son perpendiculares son 
dos procesos distintos que pueden llevar a la conclusión que "las rectas son perpendiculares", el primero se circunscribe en el paradigma GI, el segundo al paradigma GII (KUZNIAK, 2006). Para el número natural, esta distinción no aparece. Tomemos por ejemplo el criterio de divisibilidad por nueve en base diez con números de dos cifras. Un número $N$ con cifra de las unidades $u$ y decenas $d$ se escribe $N=10 d+u$. Así, $N=9 d+(d+u)$ y $N$ es divisible por 9 si y solamente si $u+d$ es divisible por 9. Para justificar el criterio, entre esta demostración y tomar todos los números de 0 a 99 no existe ninguna diferencia sobre la validez matemática. Una diferencia aparece con números de 3 o 4 cifras, pues la verificación es larga - es una variable didáctica. Pero potencialmente, para la prueba, siempre es posible hacer esta enumeración exhaustiva de todos los números de 3 o 4 cifras, posiblemente con la ayuda de la informática.

Una verdadera diferencia aparece cuando no se fija el número de cifras y, más generalmente, cuando el infinito de $\mathbf{N}$ interviene: hay que distinguir varios tipos de argumentaciones que podrían ser similares a la diferencia GI / GII. Por ejemplo, si los "ejemplos no bastan para probar" entramos en una perspectiva experimental. Se pueden hacer conjeturas y necesitan ser probadas .Seguidamente, nuevos tipos de pruebas se pueden dar como, por ejemplo, la prueba por recurrencia.

Cabe señalar, que estos aspectos no son frecuentes en la formación de profesores de primer grado, y en particular, la prueba por recurrencia no se utiliza. También, las pruebas con un número indefinido de cifras son poco frecuentes. Es posible percibir un nuevo paradigma cuando el infinito de los números naturales interviene, sin embargo, esto formará parte de nuestro estudio.

\subsection{Los registros}

Utilizamos la noción de registro de representación de Duval (1995, 2006), quien particularmente hace la diferencia cognitiva entre dos tipos de transformaciones semióticas: los tratamientos, una transformación semiótica en un mismo registro de representación, y las conversiones, una transformación semiótica cuyo resultado es expresado en otro registro. En (NIKOLANTONAKIS; VIVIER, 2010, 2013) hemos mostrado que las numeraciones en dos bases diferentes pueden ser considerados como dos registros diferentes.

El trabajo de la comunidad matemática para los números naturales se refiere de manera casi exclusiva al registro de la escritura decimal. Como el aspecto semiótico está incluido en el ETM, no podemos hablar de paradigmas aunque podríamos ver allí algunas 
características similares. Particularmente, existe una naturalización fuerte del sistema decimal con una identificación de un número con su escritura. Para estudiar cómo los profesores en formación inicial tendrán que enseñar este sistema decimal, parece pertinente "despegar" algunos elementos.

Desde el punto de vista del funcionamiento, el sistema posicional usual en una base diferente a la diez es resulta interesante debido a que los principios son idénticos a lo que los profesores tendrán que enseñar. Consideramos necesario re-aprender el funcionamiento del sistema numérico en el ámbito de la formación. Así, podemos pensar que enriquecemos el ETM-personal del profesor entorno al número natural $\left(\mathrm{ETM}_{\mathbf{N}}\right)$ con un nuevo registro de representación.

También la numeración posicional en base diferente de diez, y otros sistemas ${ }^{3}$ se utilizan en la formación de profesores, esencialmente, para representar los números naturales y mostrar algunos principios (aditivo, multiplicativo, las unidades de numeración, la base diez) pero no para hacer operaciones y desarrollar el referencial teórico.

Los tratamientos propuestos en otras bases diferente de la de diez son pobres, poco numerosos y sobre todo escasamente trabajados. Esto tiene como consecuencia la restricción a solo dos tipos de conversiones ya que sólo los cálculos en base diez se pueden efectuar:

1. Para una conversión desde la base diez, utilizamos divisiones euclidianas sucesivas por la nueva base $b$, efectuadas en base diez;

2. Para una conversión hacia la base diez, escribimos el número como un polinomio de la base inicial $b$ para luego realizar cálculos (multiplicaciones y adiciones) en base diez.

Estas conversiones utilizan artefactos simbólicos que son algoritmos. El hecho de no trabajar los cálculos en una base distinta a la diez, no permite una buena integración de estas dos conversiones en el ETM porque están en el polo artefacto sin relación con el referencial teórico. Así, la disimetría es importante y confiere sobre la base diez un estatutos matemático que no puede ser justificado, lo cual tiene consecuencias sobre el desarrollo del ETM, específicamente en el referencial teórico.

En la sección siguiente, ponemos de manifiesto los dos fenómenos enunciados en la introducción. Ambos se constituyen en obstáculos al integrar otros registros en el ETM $_{\mathbf{N}}$.

\section{Un estudio en Francia y en Grecia}

\footnotetext{
${ }^{3}$ Tradicionalmente se muestran sistemas que emergieron en la historia (egipcia, babilónica, romana..).
} 
El estudio concierne a 334 estudiantes en formación del profesorado de primer grado: 139 estudiantes de primer año de la facultad pedagógica de Florina de la universidad de Macedonia-Oeste, en Grecia, y a 195 estudiantes del centro de formación de Tours de la universidad de Orleans, en Francia. Propusimos una prueba escrita con diferentes tipos de tareas, todas relacionadas con los aprendizajes de la escuela primaria. Ver Nikolantonakis y Vivier $(2009,2010,2013)$ para una presentación más amplia.

A continuación, presentamos los resultados en porcentajes para cada una de las poblaciones y fenómenos enunciados. Para cada pregunta se describe la tarea y la tabla de los porcentajes de respuestas. En cada ítem utilizamos sistemáticamente la siguiente codificación: NR, para una no respuesta; OK, para una solución correcta; Diez, para una conversión en base diez (visible o descubierta por un análisis de error) y un tratamiento en base diez. Este último permite poner de manifiesto el fenómeno 1, y el fenómeno 2, que se asigna por variables específicas a cada ítem.

\subsection{La tarea $\underline{\text { SA: }}$ sucesores $y$ antecesores}

Encontrar al sucesor y el antecesor de: $\quad$ a. $(66)_{\text {siete }} \quad$ b. $(10100)_{\text {dos }}$ c. $(504302155)_{\text {seis }}$

Considerando las dificultades, agrupamos estas tareas en los siguientes cuatro tipos:

$\underline{\text { Sa: }}$ encontrar el sucesor de $(66)_{\text {siete }}$ con la eventual utilización de "7", y cuya respuesta posible es 67 , una utilización de la base en la escritura sin reserva, y 70, una utilización de la base en la escritura con una reserva y sin poner una tercera cifra.

$\underline{\mathbf{A b}}$ : encontrar al antecesor de (10100) ${ }_{d o s}$ cuya respuesta posible es "10099".

$\underline{\text { Sc: }}$ encontrar al sucesor de $(504302155)_{\text {seis }}$ con la eventual utilización de "6", y cuya posible respuesta son 504302156 y 504302160 al igual que $\underline{\text { Sa. }}$.

SAd: otros sucesores y antecesores no problemáticos; son tratados juntos (Aa, Sb, Ac).

Tabla 1 - Tipos de respuestas por la tarea SA, en porcentaje

\begin{tabular}{|l|c|c|c|c|c|c|c|c|c|c|c|c|c|c|c|}
\hline & \multicolumn{4}{|c|}{ Sa } & \multicolumn{4}{|c|}{ Ab } & \multicolumn{4}{c|}{ Sc } \\
\hline & NR & OK & Diez & $\mathbf{7}$ & NR & OK & Diez & $\mathbf{1 0 0 9 9}$ & NR & OK & Diez & $\mathbf{6}$ & NR & OK & Diez \\
\hline Grecia (139) & 4,3 & 87,1 & 2,2 & 5,0 & 5,0 & 72,7 & 2,9 & 1,4 & 6,5 & 84,2 & 2,2 & 2,8 & 4,3 & 87,1 & 2,9 \\
\hline Francia (194) & 8,3 & 66,0 & 3,6 & 17,5 & 14,4 & 62,9 & 4,1 & 4,6 & 11,9 & 71,1 & 3,1 & 7,8 & 14,4 & 83,5 & 5,2 \\
\hline
\end{tabular}


Los estudiantes responden con bastante éxito en este tipo de tarea, codificado $\underline{\mathbf{S A}}, \sin$ duda porque estas tareas (con bases distintas a la diez) son bien trabajadas en ambas instituciones de enseñanza (también de da éxito para el tipo de tarea de clasificación, codificado $\underline{\text { Class }}$ ).

Es probable que se use poco la base diez (entre el $2 \%$ y el $5 \%$ según los ítems) por la misma razón: el fenómeno 1 no es muy visible sobre estos tipos de tareas. En algunos estudiantes observamos el fenómeno 2 con la utilización de las cifras 7 para $\underline{\mathbf{S a}}, 6$ por $\underline{\mathbf{S c}}$, y 9 para $\underline{\mathbf{A b}}$. Este fenómeno es más visible en los estudiantes franceses con $17,5 \%$ en $\underline{\mathbf{S a}}$ y un $7,8 \%$ para $\underline{\mathbf{S c}}$.

Nosotros consideramos que existe una utilización de las propiedades (referencial teórico del ETM) en una base distinta de la diez debido al tipo de tareas empleadas.

\subsection{La tarea Once: codificación en base once}

Escribir los números de veinte a treinta en base once.

"A": utilización de una letra (o cualquiero signo), en oposición a la utilización de "10" como en (1(10) $)_{\text {once }}$ o 110; Conf: confusión número / codificación de un número.

Tabla 2 - Tipos de respuestas por la tarea Once, en porcentaje

\begin{tabular}{|l|c|c|c|c|c|}
\hline & NR & OK & Conf & “A” & “10” \\
\hline Grecia (139) & 20,1 & 22,3 & 28,8 & 12,9 & 12,2 \\
\hline Francia (194) & 15,0 & 50,5 & 26,8 & 39,2 & 16 \\
\hline
\end{tabular}

Para el tipo de tarea Once no existe mucho éxito, debido que no es trabajado, o escasamente, y los estudiantes griegos presentan más dificultades con este tipo de tareas. Una conversión en base diez no es de ninguna utilidad para Once, que corresponde a una conversión de la base diez en base once. Aquí, la distancia que hay que tomar con la base diez es grande porque hay que añadir cifras mientras que en la formación se trabaja usualmente con cifras de la base diez que se disponen. Identificamos aquí un elemento cognitivo muy importante, aspectoreñado en trabajos anteriores de Nikolantonakis y Vivier (2010, 2013).

La dificultad del tipo de tarea Once reside en el hecho que la base es estrictamente superior a diez y es necesario codificar el número diez. Los estudiantes principalmente codifican el número diez por una letra, como $\mathrm{A}, \mathrm{X}$ o $\alpha$, o bien, por su codificación en base diez, como en $(110)_{\text {once }}$, (1(10)) once o $(110)_{\text {once }}$ (todos considerados como "OK" aunque 
$(110)_{\text {once }}$ no es exactamente correcta). Es notable, que las variables "A" y "1_10" tienen roles idénticos en los grafos implicativos, y están al inicio de los árboles implicativos que contienen las variables OK. Esto significa, que tener un signo específico para designar una cifra no es importante (polo representamen) y, en cambio, la comprensión de la codificación en general (polo referencial teórico) parece ser una de las claves para resolver los problemas de tratamientos en bases distintas a la diez. Esto no es muy sorprendente pues ¿cómo podría ser fiable una técnica si no se controla el registro en el que se aplica?

\subsection{La tarea Class: clasificación}

Ordenar en forma creciente los números siguientes:

$$
(303)_{\text {cuatro }} ;(203)_{\text {cuatro }} ;(1003)_{\text {cuatro }} ;(33)_{\text {cuatro }} ;(100)_{\text {cuatro }}
$$

Tabla 3 - Tipos de respuestas por la tarea Class, en porcentaje

\begin{tabular}{|l|c|c|c|}
\hline & NR & OK & Diez \\
\hline Grecia (139) & 3,6 & 80,6 & 20,9 \\
\hline Francia (194) & 8,3 & 83,0 & 8,8 \\
\hline
\end{tabular}

Como se observa, los estudiantes respondieron con éxito esta pregunta, lo que no nos parece sorprendente, puesto que junto con $\underline{\mathbf{S A d}}$, constituyen los únicos ítems que pueden ser desarrollados correctamente aplicando solo los conocimientos de la base diez a la base cuatro, sin adaptación. Existe claramente una utilización de propiedades (referencial teórico) pero no podemos saber si se trata del fenómeno 2, porque la técnica es exactamente la misma en base diez (también para los tipos de tareas "multiplicación por ocho en base ocho", codificado $\underline{\mathbf{X 8}}$, y “determinar la paridad", codificado $\underline{\text { PIa }}$. Sin embargo, a pesar de la sencillez de este tipo de tareas se ve de manera explícita conversiones en base diez (más de $20 \%$ para la población griega): al respecto, nos preguntamos si estamos en presencia del fenómeno 1 o bien existe un control de las respuestas por parte de estos estudiantes.

\subsection{La tarea X8: multiplicación por ocho}

Escribir el resultado de la multiplicación de (3405) $)_{\text {ocho }}$ por ocho. 
Poli: escritura polinomial sin cálculo y tratamientos sobre esta escritura (nuevo registro); "27240": utilización de la técnica de multiplicación en base diez en base ocho.

Tabla 4 - Tipos de respuestas por la tarea X8, en porcentaje

\begin{tabular}{|l|c|c|c|c|c|}
\hline & NR & OK & Diez & Poli & “27240” \\
\hline Grecia (139) & 15,8 & 64,0 & 77,0 & 0,0 & 0,7 \\
\hline Francia (194) & 17,0 & 59,8 & 42,3 & 6,2 & 5,7 \\
\hline
\end{tabular}

La utilización de la base diez es casi sistemática para los estudiantes griegos. Se puede ver que la suma de los porcentajes de sus respuestas "Diez" y "NR" es cercano al 100 \%: el fenómeno 1 es importante en este tipo de tareas. También, los estudiantes franceses usan la base diez pero en menor porcentaje, y este hecho puede explicarse por la técnica que consiste a "añadir un cero", bastante frecuente, pero sin que se pueda afirmar que se trata del

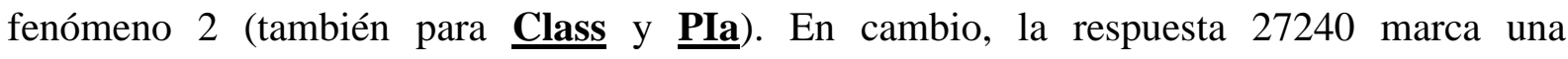
incomprensión profunda del sistema de representación (referencial teórico) y pocos estudiantes utilizan la escritura polinomial, un elemento importante del referencial teórico.

\subsection{La tarea PI: par/impar}

Para los números siguientes, decir si son pares o impares: a. (65474) ocho b. $(623004261)_{\text {siete }}$

Tec diez: Utilización de la técnica en base diez, la cual no es válida en este caso (igual a "27240" en $\underline{\mathbf{X 8}}$ ), da una respuesta correcta en el ítem a, codificado $\underline{\text { PIa }}$ pero provoca un error en el ítem b, codificado $\underline{\text { PIb}}$; Poli: escritura polinomial sin cálculo y tratamientos sobre esta escritura (igual a $\underline{\mathbf{X 8}}$ ).

Tabla 5 - Tipos de respuestas por la tarea PI, en porcentaje

\begin{tabular}{|l|c|c|c|c|c|c|c|c|c|c|}
\hline & \multicolumn{9}{|c|}{ PIa } & \multicolumn{6}{c|}{ PIb } \\
\hline & NR & OK & Diez & Tec diez & Poli & NR & OK & Diez & Tec diez & Poli \\
\hline Grecia (139) & 15,1 & 65,5 & 78,4 & 0,0 & 0,0 & 18,0 & 58,3 & 75,5 & 0,0 & 0,0 \\
\hline Francia (194) & 15,0 & 67,5 & 43,3 & 23,7 & 19,1 & 19,6 & 46,4 & 41,8 & 23,2 & 13,9 \\
\hline
\end{tabular}

Este ítem es difícil, en particular, porque la base es impar. La dificultad del ítem $\underline{\text { PIb }}$ puede explicarse por el número propuesto, que tiene un gran número de cifras, así como por la paridad de la base que hace fallida la técnica usual de la base diez. Así, si el fenómeno 1 está más presente en los estudiantes griegos (más del 75\%, casi la totalidad de los estudiantes que han respondido a estos ítems). Para los estudiantes franceses, observamos ambos fenómenos: 
más del $40 \%$ para el fenómeno 1 y casi el 25\% para el fenómeno 2 . Al igual que la tarea $\underline{\mathbf{X 8}}$, los estudiantes franceses (el $14 \%$ - $19 \%$ ) convocan la escritura polinomial (referencial teórico), lo que no es el caso de los estudiantes griegos (el 0\%). La escritura polinomial permite evitar el fenómeno 2 y puede ayudar a identificar el criterio de paridad en base impar, y con esto el referencial teórico se enriquece.

Además, observamos que la variable didáctica de $\underline{\mathbf{P I b}}$ (un número con muchas cifras) no tiene incidencia sobre el fenómeno 1 . Nos parece que este punto es relevante y refuerza la importancia de este fenómeno.

\subsection{Conclusión del estudio: ETM personal e idóneo guiados por la base diez}

Este estudio permite confirmar la fuerte presencia de ambos fenómenos en la formación inicial de profesores. Cabe señalar, que para analizar nos basamos en las trazas escritas y es probable, que ciertas respuestas dependan del fenómeno 2 sin que pudiéramos saberlo (técnica usual de clasificación y reglas de los ceros particularmente).

Ambos fenómenos muestran un tipo de desconexión de la génesis discursiva con el plano [Sem-Ins], porque la génesis discursiva proviene de un referencial teórico pobre (porque ha sido poco trabajo o simplemente no está disponible para el estudiante). En términos del ETM, no existe desarrollo ni evolución del referencial teórico (fenómeno 1), o bien, porque el referencial teórico se desarrolla pero con una importación sin control, y a veces errónea, de propiedades de la base diez (fenómeno 2). En ambos casos, los objetivos de conceptualización esperada y de enriquecimiento del ETM de los números naturales por la integración de nuevos registros parecen no ser alcanzados. El nuevo sistema semiótico cumple entonces solo una función de comunicación y no se constituye como un registro ya que no cumple las otras dos funciones cognitivas de tratamiento y de objetivación.

Para una gran parte de los estudiantes, futuros profesores, su ETM personal es guiado fuertemente por la base diez y el ETM idóneo propuesto no permite desarrollar un ETM operacional para bases distintas de la de diez. El usar otras bases a la de diez, permite desplegar un ETM idóneo en el cual los números se representen de otra manera pero sin hacer tratamientos usuales de la aritmética. Estas nuevas representaciones no son asociadas a conocimientos teóricos porque la relación no es espontánea (excepto para la tarea de clasificación). Entonces, podemos pensar que no proponemos un nuevo registro a los 
estudiantes (futuros profesores) en que ellos sean capaces de desarrollar su ETM personal, ya sea porque no existen, o bien, porque son escasos los tratamientos.

El registro en numeración decimal, de cierta manera tiene características próximas de un paradigma y guía el trabajo matemático. Entonces ¿una codificación en otra base de la de diez representa un número? Podemos considerar que no lo es para muchos estudiantesprofesores.

Existe también una diferencia entre las dos poblaciones. Los estudiantes griegos tienen

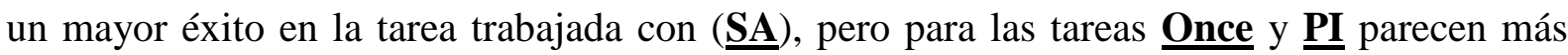
desestabilizados que los estudiantes franceses. Esto nos recuerda la diferencia de flexibilidad entre las dos poblaciones en geometría (NIKOLANTONAKIS; VIVIER, 2015).

Sin embargo, consideramos que es posible intervenir a nivel del ETM idóneo. Por una parte, proponiendo tareas más ricas en bases distintas que la base diez, y de otra parte, apoyándose sobre todo en la escritura polinomial. La escritura polinomial permitiría evitar el fenómeno 2 y enriquecer el referencial teórico. No obstante, esto último no fue la elección realizada y en la sección siguiente se desarrolla una otra propuesta.

\section{Una situación para tomar en consideración el referencial teórico}

Con el fin de tomar en consideración el referencial teórico, exploramos un camino utilizando la situación de Tempier (2013). En este trabajo se desarrolla una situación de ingeniería colaborativa con profesores de CE2 en Francia (grado 3) apoyándonos en los dos principios fundamentales de la numeración posicional usual.

Se ha realizado una experimentación (anexo 1) para que las nuevas representaciones de los números naturales en bases distinta a la base diez sean operacionales, y también, para enriquecer el referencial teórico del ETM. Se ha incorporando el registro de base siete $^{4}$ para reparar en la falta que ha sido identificada. Así, el objetivo es proponer una situación donde las tareas se efectuaran en base siete (y evitar el fenómeno 1) desarrollando el referencial teórico mediante la base siete (y evitar el fenómeno 2).

Participaron 28 estudiantes de tercer año de licenciatura pluridisciplinaria de la universidad París Diderot. Los dos tercios de ellos siguen la opción Profesor de Escuela y un tercio sigue la opción de Mediación Científica. La mayoría de ellos no han seguido estudios

\footnotetext{
${ }^{4}$ La elección de la base responde a varias condiciones: tener una base inferior a diez, tener al menos tres cifras, sin utilizar dos cifras de la base diez, y tener bastantes diferencias con la base diez. Podíamos elegir entre la base seis, como en el estudio de Anselmo y Zucchetta (2013), y la base siete.
} 
de matemáticas en la universidad y, por la opción Profesor de Escuela, no son del área de ciencias. Los estudiantes, con la excepción de uno de ellos, no conocen las bases de numeración distinta a la diez.

El trabajo de los estudiantes se organizó en nueve equipos y se les estregó a cada uno las preguntas en papel; para codificar se usaron las iniciales de los estudiantes. Presentamos a continuación los resultados a las preguntas, a excepción para la tarea E) Conversiones, porque no hay ningún problema (muy buen éxito) y para F) Síntesis, porque las respuestas son débiles (estas tareas aparecen en el anexo 1).

\subsection{Aspecto ordinal}

Las respuestas dispuestas en una tabla no fueron completadas correctamente por ningún equipo en una primera fase y todos propusieron 36 37, o bien 6670 (cf. los resultados de 3.1). También, se encontraron respuestas como 81, 91 y $99 \mid 100$. A pesar de la evidente diferencia con la base diez en la escritura de los primeros números, las secuencias propuestas hacen directa referencia al sistema decimal y en diferentes niveles (fenómeno 2), como si existiera una dificultad en salir del paradigma de la base diez. Una intervención colectiva (prevista) se realizó para que cada equipo pudiese avanzar. Después de esta discusión en común, en una segunda fase todas las respuestas fueron correctas. Hay referencias a la base diez como en la descripción de las cifras ("de 0 a 6" o "7, 8 y 9 no existen") y utilizaciones de "decenas", "decena superior" o "centena" (creemos que sobre todo aquí existe una falta de vocabulario). El equipo AJFB re-nombra todas las cifras y elabora las tablas de adición (cf. la figura 1) como si se quisiera rechazar referencias a la base diez. En cambio, el equipo SML dice: "para cambiar de casilla, es necesario sumar +1 y cuando es 6 es necesario sumar +4 ". Aunque la tabla es correcta, hay un problema en el sentido pues ¡algunos sucesores son obtenidos sumando +4 ! El trabajo de este equipo se realizó básicamente en el plano [Sem-Ins], pues la naturaleza de los objetos representados, los números naturales, y sus propiedades están ausentes, y el referencial teórico está totalmente subordinada a la base diez.

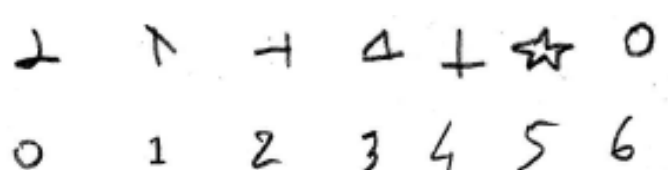




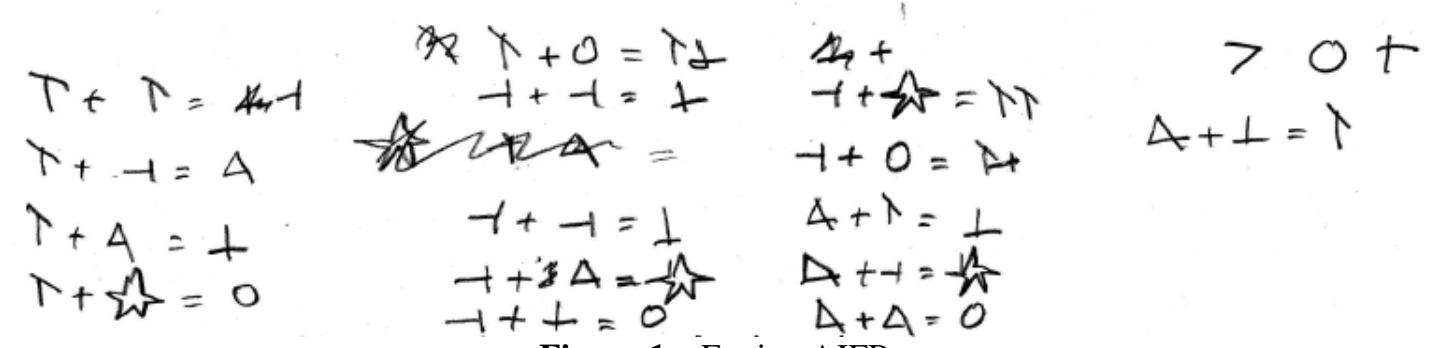

Figura 1 - Equipo AJFB

Las respuestas de los estudiantes al comparar fueron logradas por todos los equipos, a veces con marcas (signos) de conversión en base diez (fenómeno 1). Encontramos los mismos tipos de respuestas que para el estudio precedente al tipo de tarea Class con la siguiente justificación: "comparación de los valores decimales como en un sistema clásico", "la misma comparación que para el sistema decimal" o "la misma manera". Así como para Class, no se puede saber si se trata del fenómeno 2 .

\subsection{Aspecto cardinal}

A las preguntas relativas a la enumeración de una colección para determinar su cardinal $^{5}, 8$ de 9 equipos entregaron un buen resultado, de ellos 6 equipos contaban las bolas con paquetes de 7, más o menos explícitamente. Un equipo afirma "¡agrupamos los “o" por equipos de 10!" - el signo de exclamación es esencial - este equipo procedió contando oralmente: “uno, dos,..., seis, diez, once,..., dieciséis, veinte, veintiuno..." y entregaron así el buen resultado cifrado. Se observa, que ellos utilizaron el registro de la lengua natural asociada a la base diez. En este caso, nos parece difícil referirnos del fenómeno 1 o 2, puesto que más bien, vemos una falta de vocabulario asociado a la base siete. Lo mismo ocurre con otro equipo que explica "contamos hasta seis muchas veces y pasamos a la...", nosotros pensamos que a este equipo les faltan palabras y no saben cómo expresarse.

En tres equipos encontramos la presencia del fenómeno 1, ellos cuentan oralmente en base diez para luego utilizar la tabla como ábaco. Un equipo (IOW) lo utilizó al revés: el número "treinta y seis" es 36 en base diez que es en la casilla vigésimo-séptimo pues da la codificación 27 (también anotamos aún la utilización del signo 7). El trabajo de este equipo es únicamente en el plano [Sem-Ins], sin control semántico que podría aportar el referencial

\footnotetext{
${ }^{5}$ En el primer cuadro (cf. anexo 1) es $36=51_{\text {sept }} \mathrm{y}$ en el segundo es $33=45_{\text {sept }}$.
} 
teórico; se trata de un juego de codificación (semiótica) instrumentado por la tabla (con errores).

Además, se encuentran explicaciones de conversión (Figuras $2 \mathrm{a}$ y $2 \mathrm{~b}$ ) que nos permiten pensar que para estos equipos existe una buena integración del nuevo registro en el $\mathrm{ETM}_{\mathbf{N}}$ con un desarrollo del referencial teórico.

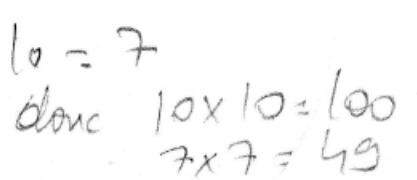

Figura 2a - Equipo IOW

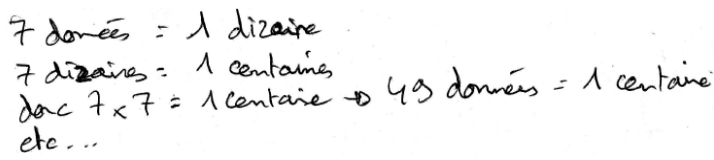

Figura 2b - Equipo SML

\subsection{Unidades de numeración y escrituras cifradas}

En la escritura de $u_{k}$ para $k=5,6,10,11$ y 12 , hay 7 respuestas correctas (esperadas) y dos respuestas $u_{10}=10000000000$ en lugar de 10000000 (ídem para $u_{11}$ y $u_{12}$; el equipo MFML propuso esto como primera intención antes de borrarlo y de responder correctamente). Encontramos aquí la presencia del fenómeno 2, con la asociación casi-automática entre la codificación 10 y el número diez.

También, encontramos, escrituras interesantes que permiten ir más lejos en la comprensión de este nuevo sistema como $7^{k}$ y $(6+1)^{k}$ y $u_{10}=(6+1)^{6+1}$ (equipo SML), $u_{100}=10^{100}$ (equipo OSM) y $u_{k}=10^{k}$ que posteriormente fue borrado (equipo MFML). Así, aunque esta pregunta tenía solo como objetivo introducir una notación para las unidades de numeración, nos parece también una oportunidad para el desarrollo del referencial teórico.

En cuanto a la búsqueda de las escrituras cifradas encontramos 8 respuestas correctas. Sin duda, se encuentran unas posiciones por rango (con añadido de cero si es necesario) pero hay pocas marcas visibles. Sin embargo, posiblemente para justificar, vemos tablas de numeración (MCC y SML, ver figura 3) y cálculos correctos que se apoyan en un registro próximo de la escritura polinomial como: "4000+500+2=4502", "4000; $500 ; 2 \rightarrow 4502$ " o

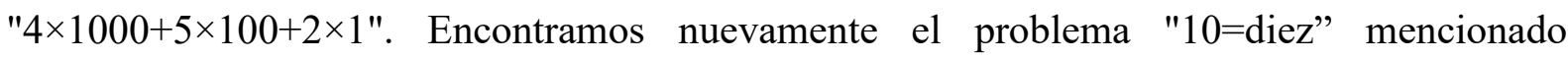
anteriormente pero que contienen errores como $4 \times 10=55$ o $5 \times 10=101$ (la explicación proviene de $40_{\text {diez }}=55_{\text {siete }}$ y $50_{\text {diez }}=101_{\text {siete }}$ ). 


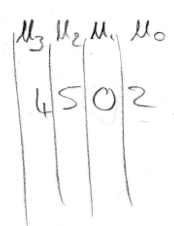

Figura 3 - Equipo MCC

\subsection{Sumas}

En este caso, encontramos cuatro respuestas correctas y un equipo no respondió. El fenómeno 2 es presente en dos equipos (RLC e IOW) que hacen adiciones en base diez (respuestas 425, 4무 $3, \underline{\mathbf{9}} 6,46 \underline{\mathbf{9}} 3$ ).

Entre las respuestas correctas encontramos:

1. Conversiones directas en la suma bruta con escrituras transitorias que utilizan los signos 7, 8 y 9 (figura 4) - Aunque se apoya en la base diez, no está presente el fenómeno 2 porque existe un control de los cálculos efectuados;

2. Un trabajo con reservas en las adiciones (figuras 5a y 5b) - observamos una integración del nuevo registro en el $\mathrm{ETM}_{\mathbf{N}}$.

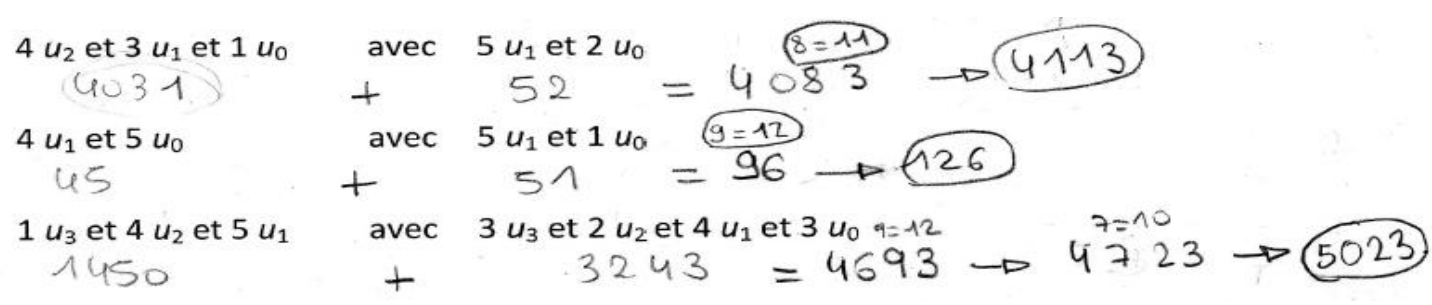

Figure 4 - Equipo LM

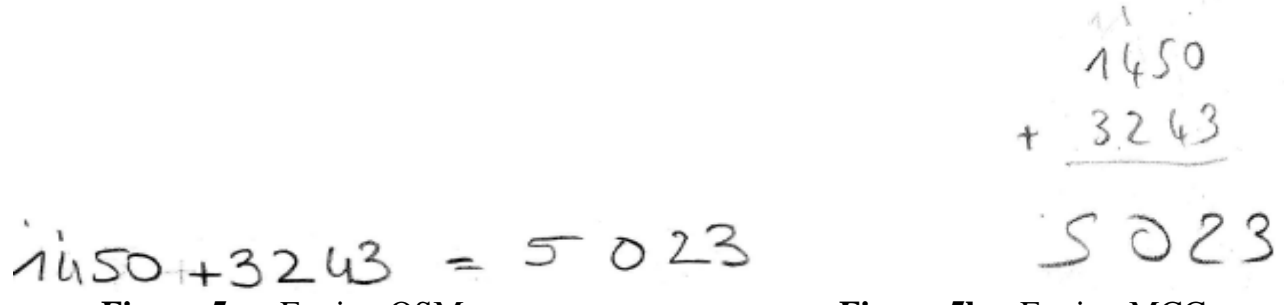

Figura 5a - Equipo OSM

Figura 5b - Equipo MCC

Además, se observa que un equipo efectúa la adición y utiliza las reservas pero con una mala gestión porque traslada la suma de ambas cifras. Consideramos que esta respuesta no obedece a la presencia del fenómeno 2, sino más bien, que el referencial teórico está en proceso de constitución. 


\subsection{Conclusión de la sección 4}

Los objetivos de esta situación se alcanzan en el sentido que la mayoría de los equipos descubren y utilizan nuevos conocimientos en base siete, pero apoyándose en la base diez. Este apoyo nos parece esencial, porque esta misma población no tuvo éxito cuando se les propuso, a la semana siguiente, la misma situación pero con el sistema posicional de Zeckendorf cuyas unidades de numeración son los números de la secuencia de Fibonacci. Pero, para esta población, la distancia con la base diez es demasiado grande y la situación plateada no tuvo éxito y debió interrumpirse. Los objetivos trazados no fueron logrados.

No obstante, el fenómeno 1 (conversión en base diez) siempre está presente, sin duda porque trabajar en una base usual tranquiliza a los estudiantes. Es difícil pensar en una situación que permita el apoyo sobre los conocimientos de la base diez y, al mismo tiempo, impida las conversiones en base diez para efectuar las tareas.

Para el fenómeno 2 (utilización de las propiedades de la base diez en otra base), podemos distinguir varias maneras de utilizar la base diez:

1. Una utilización lingüística, como "decena" o "centena" para designar las codificaciones "10" y "100" de la base de numeración o la secuencia de los números "uno dos... seis, diez, once,..." que proviene de una falta de vocabulario adaptado;

2. Una utilización de las cifras para escribir los números como $36 \mid 37$ y $66 \mid 70$, lo que puede tener consecuencias en los cálculos ulteriores;

3. Una utilización sin control de los conocimientos de la base diez que afecta el sentido de los objetos matemáticos, como en "10=diez", "sumar +4 para tener el sucesor" (ver también las secciones 3.4 y 3.5);

4. Una utilización transitoria de las cifras, como en " $5+3=8$ " y " $8=11$ " con la reserva 1 ;

5. Una utilización mixta de dos registros como en "49=1 centena" o "100=49", un trabajo donde puede emerger nuevos conocimientos;

6. Una utilización intrínseca para la nueva base como $u_{10}=(6+1)^{6+1}$ o $u_{k}=10^{k}$ que permite una integración del nuevo registro en el referencial teórico.

La utilización de 1 y 2 son sintácticas y no afectan el sentido de los objetos matemáticos, pero con esto el referencial teórico no se desarrolla. También, la utilización de 3 
es sintáctica pero, aunque es operacional, afecta el sentido y esto puede tener incidencias nefastas sobre el desarrollo del referencial teórico. Las utilizaciones de 4 y 5 se apoyan en la base diez para producir nuevos conocimientos y desarrollan el referencial teórico, pero la ambigüedad de la base utilizada puede ser una fuente de complicaciones y una reformulación es necesaria para acercarse a la utilización 6 que es exactamente lo esperado.

\section{Conclusiones}

La primera experimentación permitió poner de manifiesto ambos fenómenos relacionados a la introducción de los nuevos registros constituidos por la numeración en bases distintas a la base diez para los números naturales. Utilizamos una experimentación de (NIKOLANTONAKIS; VIVIER, 2009) que se estudió con Análisis Estadístico Implicativo y un marco de análisis didáctico que consideraba los registros de representación de Duval (1995, 2006) y las praxeologías de Chevallard (1999). En esta investigación, se concluyó que los sistemas numéricos pueden ser considerados como registros diferentes, el potencial tecnológico del registro de la escritura polinomial, y la jerarquía de los tipos de tareas según la base de numeración: Once $e_{a>d i x}-\mathrm{PI}_{a \text { impair }}-\mathrm{X}_{a \neq d i x}-\mathrm{PI}_{a \text { pair }}-\mathrm{PS}_{a \neq d i x}-\mathrm{Class}_{a \neq d i x}$.

En cuanto al uso de la base diez, habíamos identificado el débil desarrollo de los nuevos registros que consistía en convertir en base diez para efectuar la tarea, lo que corresponde al fenómeno 1. Sin embargo, no habíamos utilizado el modelo de los ETM y lo que aparece gracias a este modelo, es la naturaleza del trabajo realizado, particularmente el fenómeno 2 que no habíamos claramente identificado en las investigaciones precedentes. Nos parece que se comprende mejor el sistema en juego y lo que se podría hacer con el fin de evitar ambos fenómenos, los cuales impiden el objetivo de conceptualización, es decir, el desarrollo del $\mathrm{ETM}_{\mathbf{N}}$.

Por otra parte, los estudiantes que ingresan a la formación de profesores de escuelas primarias son totalmente novatos en el tema de la numeración posicional en base distinta a la de diez. Sus ETM $_{\mathbf{N}}$ personales tienen que construirse en totalidad, en apoyo con sus ETM $_{\mathbf{N}}$ iniciales que únicamente hacen referencia a la base diez. La base diez constituye un apoyo fundamental, pero nos parece se debe tomar distancia con la base diez con el fin de evitar la aparición de ambos fenómenos. Así, es esencial cuestionar el $\mathrm{ETM}_{\mathbf{N}}$ idóneo propuesto, o bien, preguntarse ¿cómo construir un $\mathrm{ETM}_{\mathbf{N}}$ idóneo para integrar las numeraciones en otras bases diferentes de la de diez? Al respecto, hemos identificado las siguientes dos pistas: 
1. El registro de la escritura polinomial: su potencial para el desarrollo del referencial teórico es muy importante, a pesar que este registro no es muy utilizado;

2. La situación de Tempier (2013): sea adaptada en base siete con el fin de centrar el trabajo sobre los principios de estos sistemas de numeración.

Cabe señalar, que solo la segunda situación ha sido estudiada en esta investigación. Evidentemente no se trata de una solución ideal, hay equipos que tienen dificultades. Pero los resultados son prometedores y, para la mayoría de los equipos, los conocimientos relativos a la base diez se utilizan con una reinterpretación en la nueva base. Además, es exactamente el objetivo que se da en la formación de profesores para la numeración de los números naturales, teniendo como perspectiva que serán ellos, como futuros profesores, quienes tendrán que enseñar la numeración decimal.

Esta investigación debe continuar y mejorarse, particularmente, se debe aumentar el tiempo de la situación para fortalecer los conocimientos. Además, y tal como lo hemos mostrado, la situación de aprendizaje puede ser iniciada con éxito con la situación de Tempier. El desafío es continuar y mejorar nuestro trabajo pero incorporando el registro de la escritura polinomial.

Es necesario considerar que el problema no es especifico de los números naturales y de la numeración posicional. El tema investigado concierne a todos los registros que son pocos (o no son) utilizados para efectuar tratamientos, cuestión que también lo hemos evidenciado pero con los números racionales. Al respecto, podemos señalar, que el registro de la escritura decimal (infinito periódico) no permite hacer tratamientos, excepto comparaciones y cálculos simples (y no justificados). Así es señalado por Rittaud y Vivier que proponen algoritmos para efectuar las operaciones de bases en el registro decimal, lo que permite trabajar el sistema semiótico como un registro y realizar tratamientos. En particular, un trabajo con la adición ha sido utilizada en la formación de profesores de segundo grado (para este tema, ver (VIVIER; 2015) y (RITTAUD; VIVIER, 2016)).

\section{Referencias}

ANSELMO, B.; ZUCCHETTA, H. Du comptage à la numération - une formation sur l'enseignement de la numération, Grand N, Grenoble, v. 91, p.71-91, 2013.

CHEVALLARD Y. L'analyse des pratiques enseignantes en théorie anthropologique du didactique, Recherches en Didactique des Mathématiques, Grenoble, v.19, n.2, p. 222-265, 1999.

DUVAL, R. A cognitive analysis of problems of comprehension in a learning of mathematics, Educational Studies in Mathematics, Netherlands, v.61, n.1-2, p. 103-131, fev. 2006. 
DUVAL, R. Quel cognitif retenir en didactique des mathématiques ?, Recherches en Didactique des Mathématiques, Grenoble, v.16, n.3, p.349-382, 1996.

DUVAL, R. Sémiosis et pensée humaine. Berne : Peter Lang, 1995.

KUZNIAK, A. Paradigmes et espaces de travail géométriques. Éléments d'un cadre théorique pour l'enseignement et la formation des enseignants en géométrie. Canadian Journal of Science and Mathematics Education, Toronto, v. 6 n. 2. p 167-188, jan. 2006.

KUZNIAK, A. L'espace de Travail Mathématique et ses genèses. Annales de didactique et de sciences cognitives, Strasbourg, v. 16, p. 9-24, 2011.

KUZNIAK, A.; RICHARD, P. R. Espacios de trabajo matemático. Puntos de vista y perspectivas, Revista latinoamericana de investigación en matemática educativa, Mexico, v.17, n.4-I, p. 5-15, dez. 2014.

MONTOYA DELGADILLO E.; VIVIER, L. Mathematical working spaces as an analyzing tool for the teaching and learning of calculus., ZDM, Cham, v.48, n.6. 2016. Trabajo acceptado.

NIKOLANTONAKIS, K.; VIVIER, L. La numération en base quelconque pour la formation des enseignants du premier degré en France et en Grèce. Une étude articulant registres et praxéologies, in Chypre et France. In : GAGATSIS, A. et al. (Ed.). Recherche en didactique des mathématiques. Lefkosia : University of Cyprus, 2009, p. 171-186.

NIKOLANTONAKIS, K.; VIVIER, L. Registres et praxis pour la numération de position en base quelconque - une étude statistique en France et en Grèce. In : RÉGNIER, J. C.; et al (Ed.). Analyse statistique implicative - objet de recherche et de formation en analyse de données, outil pour la recherche multidisciplinaire. Actes du $5^{\mathbf{e}}$ colloque A.S.I., Palermo:Università degli studi di Palermo 2010, 273-288.

NIKOLANTONAKIS, K.; VIVIER, L. Positions numeration in any base for future elementary school teachers in France and Greece: one discussion via registers and praxis. Menon, Florina, v.2 n.a, p. 99114, jul. 2013.

NIKOLANTONAKIS, K.; VIVIER, L. Espaces de travail géométrique en formation initiale de professeurs du premier degré en France et en Grèce lors d'une démarche de preuve, Revista latinoamericana de investigación en matemática educativa, Mexico, v.17, n.4-I, p. 191-210, dez. 2014.

RITTAUD, B.; VIVIER, L. La mystérieuse égalité $0,9999 \ldots=1$ : regards didactiques, mathématiques et historiques. Epistémologie et didactique, Presses Universitaires de France-Comté. 2016. "Travail en cours d'impression".

TEMPIER, F. La numération décimale à l'école primaire. Une ingénierie didactique pour le développement d'une ressource. 2013. 427 páginas. Tese de Doutorado em Didática das matemáticas. Université Paris Diderot, Paris, 2013.

VIVIER, L. Sur la route des réels. Point de vue sémiotique, praxéologique, mathématique. 2015. 104 páginas. Note de synthèse (Habilitation à diriger les recherches-HDR), Paris, Université Paris Diderot. 2015.

Submetido em Julho de 2015. Aprovado em Setembro de 2015. 


\section{Anexo 1 : la situación ${ }^{6}$ de la base siete}

\section{Une nouvelle façon d'écrire les entiers naturels - Une nouvelle numération de position}

3. TOUS les nombres de cette fiche sont écrits dans ce nouveau système.

4. TOUTES les réponses de cette fiche sont à écrire dans ce nouveau système.

5. Des exemples sont proposés, vous pouvez en ajouter d'autres si vous le désirez.

\section{A) La suite des écritures chiffrées}

Continuer la suite des nombres de ce tableau (lecture de gauche à droite et de haut en bas).

\begin{tabular}{|l|l|l|l|l|l|l|l|l|l|}
\hline 1 & 2 & 3 & 4 & 5 & 6 & 10 & 11 & 12 & 13 \\
\hline 14 & 15 & 16 & 20 & 21 & 22 & 23 & 24 & 25 & 26 \\
\hline 30 & 31 & & & & & & & & \\
\hline & & & & & & & & & \\
\hline & & & & & & & & & \\
\hline & & & & & & & & & \\
\hline
\end{tabular}

Comment faire pour trouver l'écriture chiffrée du successeur d'un nombre dans ce système?

Comparer les nombres suivants (avec $>$ et $<$ selon le cas) :
$12 \ldots 4$
$35 \ldots 42$
$100 \ldots 64$
$3240 \ldots 44$
$200 \ldots 300$
$241 \ldots 256$

Comment faire pour comparer deux nombres dans ce système?

\section{B) Dénombrement d'une collection}

Combien y a-t-il de « $\mathrm{O} »$ dans ces deux cadres ? Donner le nombre dans le nouveau système.

\begin{tabular}{|c|c|}
\hline 00000000000 & O 000 \\
\hline
\end{tabular}

$\begin{array}{lllllllllllllllllll}0 & 00 & 0 & 0 & 0 & 000000000 & 00 & 0 & 0\end{array}$
Oo0 o o 000 o o o o 0000

o o o o 0000000 o 0 o 000 o

Comment faire pour dénombrer une collection avec ce système d'écriture des nombres?

\footnotetext{
${ }^{6}$ El original fue en cuatro páginas, con espacios para poder directamente responder sobre las hojas. Las partes E) y F) no están en el anexo.
} 
C) On définit les unités relatives à chaque rang de la manière suivante :

$$
u_{0}=1 ; u_{1}=10 ; u_{2}=100 ; u_{3}=1000 ; u_{4}=10000 ; \text { etc. }
$$

Écrire les unités relatives aux rangs 5, 6, 10, 11 et 12.

Comment s'écrit l'unité de rang $k$, notée $u_{k}$ ?

Trouver l'écriture chiffrée des nombres suivants :

6. $1 u_{2}$ et $4 u_{1}$ et $3 u_{0}$

7. $4 u_{3}$ et $5 u_{2}$ et $2 u_{0}$

8. $5 u_{1}$ et $2 u_{2}$ et $1 u_{0}$ et $4 u_{3}$

9. $4 u_{2}$ et $1 u_{3}$ et $5 u_{1}$

Comment faire pour trouver l'écriture chiffrée d'un nombre donné avec les unités de rang?

D) On ajoute deux nombres donnés avec les unités de rang.

Trouver les écritures chiffrées.

10. $1 u_{2}$ et $2 u_{1}$ et $3 u_{0}$
avec $3 u_{2}$ et $2 u_{0}$
avec $5 u_{1}$ et $2 u_{0}$
avec $5 u_{1}$ et $1 u_{0}$
avec $3 u_{3}$ et $2 u_{2}$ et $4 u_{1}$ et $3 u_{0}$

11. $4 u_{2}$ et $3 u_{1}$ et $1 u_{0}$

12. $4 u_{1}$ et $5 u_{0}$

13. $1 u_{3}$ et $4 u_{2}$ et $5 u_{1}$

Comment faire pour ajouter deux nombres dans ce système ? 